\title{
Immunogenicity and Protective Efficacy of a Polyvalent DNA Vaccine against Human Orthopoxvirus Infections Based on Smallpox Virus Genes
}

\author{
Rinat A. Maksyutov, Elena V. Gavrilova, Galina V. Kochneva, and Sergei N. Shchelkunov \\ State Research Center of Virology and Biotechnology VECTOR, Koltsovo, Novosibirsk 630559, Russia \\ Correspondence should be addressed to Rinat A. Maksyutov; maksrinat@yandex.ru
}

Received 26 May 2012; Accepted 11 July 2012

Academic Editor: Barbara Schnierle

Copyright (c) 2013 Rinat A. Maksyutov et al. This is an open access article distributed under the Creative Commons Attribution License, which permits unrestricted use, distribution, and reproduction in any medium, provided the original work is properly cited.

DNA vaccines combining plasmids carrying the variola virus genes M1R, A30L, and F8L of intracellular virion surface membrane proteins as well as A36R and B7R of the extracellular virus envelope proteins under control of Rous sarcoma virus or cytomegalovirus promoters have been constructed. These DNA vaccines induced production of a high titers of vaccinia virus-neutralizing antibodies in mice similar to those elicited by the live vaccinia virus immunization. Mice vaccinated by created DNA vaccine were completely protected against a lethal $\left(10 \mathrm{LD}_{50}\right)$ challenge with highly pathogenic ectromelia virus. These results suggest that such vaccine should be efficient in immunization of humans against smallpox.

\section{Introduction}

The historically first method used for human protection against devastating smallpox epidemics was the so-called variolation, that is, an intracutaneous inoculation of healthy persons with infectious material from human smallpox cases. The disease thus induced had a shorter incubation period and a milder course as compared with the disease caused by a common respiratory transmission of this infection. The mortality rate after variolation was $0.5-2 \%$ versus $20-30 \%$ typical for the smallpox epidemics. The discovery of human vaccination using first cowpox virus (CPXV) and then vaccinia virus (VACV) had led to a considerable decrease in the severe side reactions $[1,2]$.

Taking into account the postvaccination complications caused by classical live vaccine involving VACV and confirmation of the global smallpox eradication, the World Health Organization recommended in 1980 to stop further vaccination against this infection [1]. Subsequent worldwide cessation of the vaccination against smallpox has created a most dangerous situation when the human population year by year becomes ever more unprotected not only from a potential infection with variola virus (VARV) as a result of a bioterrorism attack or reemergence of the virus in nature, but also from infection with other closely related orthopoxviruses, the natural reservoir of which is small rodents [3-5]. This is demonstrated by more frequent outbreaks of human orthopoxvirus infections caused by monkeypox virus (MPXV), CPXV, and VACV [6-10]. The absence of efficient antivirals makes the vaccine prevention the most important specific tool for control of the orthopoxvirus infections among humans.

Use of the classical live VACV vaccine for mass vaccinations is now unacceptable because of a relatively large number of potential complications, especially taking into account the increased number of population cohorts with suppressed immunity, such as the patients after transplantation, HIV infected persons, and those taking immunodepressants. Correspondingly, it is especially topical challenge to design stateof-the-art safe vaccines against smallpox and other human orthopoxvirus infections. A promising direction is creation of a DNA vaccine, the newest approach to immune prevention of virus infections [11-13]. Such vaccines are able to induce a full-fledged immune response to a viral infection being completely safe for humans. 
Although the viruses belonging to the genus Orthopoxvirus are closely related and the vaccination of animals or humans with a member of this genus provides protection against infection with other orthopoxvirus species, it is known that the persons that had smallpox acquire a lifelong immunity, whereas the vaccination with vaccinia virus requires repeated immunizations with a certain periodicity to ensure no subsequent decline of immunity against smallpox $[1,3]$.

The accumulated data on the main orthopoxvirus protective virion proteins and genome sequences have formed the background for designing a polyvalent DNA vaccine with a combination of virus genes [3,14]. A considerable success in development of the DNA vaccines against orthopoxvirus infections has been demonstrated in several works where the mixtures of two-to-five plasmids carrying individual VACV genes have been used for vaccination and the protective effect has been assessed in the mice challenged with a lethal VACV dose [15-17]. A study of a combined DNA vaccine involving four VACV genes using a macaque rhesus model challenged with MPXV after vaccination has demonstrated an incomplete protective effect [18]. This suggested that use of the genes of the corresponding orthopoxvirus in DNA vaccines could provide a better protection from the orthopoxvirus infection. Consequently, the DNA vaccine variants and subunit protein vaccine based on MPXV genes have been designed and tested in a macaque rhesus model [19]. In this case, a protective effect from the lethal infection of the animals with MPXV was detected after joint administration of both the DNA and protein vaccines.

Other authors have recently synthesized codon-optimized variants of three VARV genes for an anti-smallpox vaccine [20]; however, immunization of mice with such vaccine failed to provide a complete protection from a lethal challenge with VACV.

The goal of this work was to elaborate a combined DNA vaccine based on five natural VARV genes and to study its protective efficacy in the model of mice infected with ectromelia virus (ECTV), highly virulent for these animals.

\section{Materials and Methods}

2.1. Viruses and Cells. E. coli strain XL2-Blue, culture of African green monkey kidney epithelial cells Vero, vaccinia virus strain LIVP (VACV-LIVP), and ectromelia virus K1 strain (ECTV-K1) were obtained from the collection of the State Research Center of Virology and Biotechnology Vector.

2.2. Construction of DNA Vaccines. The individual VARV genes A30L, F8L, and M1R of intracellular mature virus (IMV) and B7R, F8L of extracellular enveloped virus (EEV) were PCR amplified from VARV India-1967 DNA [21] using Taq DNA polymerase with the primer pairs listed in Table 1. Amplicons were cut with AsuNHI and SalI restriction endonucleases and cloned into an AsuNHISall site of the mammalian expression vector pBKRSV (Stratagene, USA) under the control of Rous sarcoma virus (RSV) promoter. Then, all five genes were cut with
Asu NHI and HindIII restriction endonucleases and recloned into an AsuNHI-HindIII site of the mammalian expression vector pcDNA3.1/Myc-His(-)/lacz (Invitrogen, USA) under the control of cytomegalovirus (CMV) promoter. Each construct was confirmed by restriction digestion and DNA sequencing using a 310 Genetic Analyzer (Applied Biosystems, USA). DNAs of the plasmids pcDNA, pcDNAA30L, pcDNA-F8L, pcDNA-M1R, pcDNA-A36R, pcDNAB7R, pBKRSV, pBKRSV-A30L, pBKRSV-F8L, pBKRSVM1R, pBKRSV-A36R, and pBKRSV-B7R were prepared in bulk using an EndoFree Plasmid Giga Kit (Qiagen, Germany) and suspended in sterile PBS. The DNA concentrations were determined by UV spectroscopy using an Ultrospec 3000 pro (Biochrom, UK) spectrophotometer.

2.3. Administration of DNA Vaccines. Six-week-old female $\mathrm{BALB} / \mathrm{c}$ mice were each immunized (intracutaneously, subcutaneously, intraperitoneally, or intramuscularly) with $100 \mu \mathrm{g}$ of endotoxin-free plasmid DNA in $100 \mu \mathrm{L}$ of PBS (in the case of a plasmid mixture, $50 \mu \mathrm{g}$ of each plasmid with a total dose of $250 \mu \mathrm{g}$ were used). The vaccines were administered three times with 3-week intervals; blood samples were taken 2 weeks after immunization. Animal experimentation guidelines were followed in animal studies.

2.4. Plaque Reduction Neutralization Assay. The sera from immunized animals (1/100 dilutions) were incubated with $100 \mathrm{PFU}$ (plaque forming units) of VACV-LIVP for $1 \mathrm{~h}$ at $37^{\circ} \mathrm{C}$. Confluent Vero cell monolayers were infected with antibody-virus mixtures for $1 \mathrm{~h}$, washed with PBS, and incubated under liquid overlay for 3 days. After removing the liquid, the monolayers were stained with $0.2 \%$ crystal violet for $10 \mathrm{~min}$ to count the plaques. The neutralization was calculated as the percent of the plaque counts, reduced in the tests with serum, relative to the number of the plaques in the virus control (without sera).

2.5. Challenge Experiment. After three vaccine doses were administered at intervals of 3 weeks, mice were intraperitoneally challenged with $10 \mathrm{LD}_{50}\left(3.2 \times 10^{2} \mathrm{PFU}\right)$ of ECTV$\mathrm{K} 1$. The challenge took place 3 weeks after the last immunization. The mice were observed daily during 2 weeks. Animal experimentation guidelines were followed in animal studies.

\section{Results}

3.1. The Need in Using VARV Genes for Construction of DNA Vaccine. At the first stage of the work, we compared the antigenic portraits of the VARV virion proteins A30L, A36R, B7R, F8L, and M1R and their VACV, CPXV, MPXV, and ECTV orthologs. For this purpose, we aligned the amino acid sequences of the mentioned proteins of VARV strains India-1967 [22], Bangladesh-1975 [23], and Garcia-1966 [24], MPXV strains Zaire-1996 [25], and USA_2003_039 [26]; CPXV strains GRI-90 [27] and Brighton-Red [28]; VACV Copenhagen [29], Ankara [30], and WR [31]; ECTV strains Moscow [32] and Naval [33]. The potential antigenic determinants (epitopes) of the studied proteins were 
TABLE 1: Primers used to amplify VARV genes by PCR.

\begin{tabular}{|c|c|c|c|}
\hline VARV gene & Primer sequence & Amplicon size (bp) & DNA vaccine \\
\hline $\mathrm{A} 30 \mathrm{~L}$ & $\begin{array}{c}5^{\prime} \text {-CCCGGCTAGCCGCCACC ATGGACGGAACTCTTTTCCCTG-3' } \\
5^{\prime} \text {-CCCGTCGACGTTACTCATATGGGCGCCGAC- } 3^{\prime}\end{array}$ & 360 & $\begin{array}{l}\text { pBKRSV-A30L } \\
\text { pcDNA-A30L }\end{array}$ \\
\hline A36R & $\begin{array}{c}5^{\prime} \text {-CCCGGCTAGCCGCCACC ATGATGACACCAGAAAACGAC- } 3^{\prime} \\
5^{\prime} \text {-CCCGTCGACTTAGTTCATTGTTTTAACACAAAAAT- } 3^{\prime}\end{array}$ & 581 & $\begin{array}{l}\text { pBKRSV-A36R } \\
\text { pcDNA-A36R }\end{array}$ \\
\hline B7R & $\begin{array}{c}5^{\prime} \text {-CCCGGCTAGCCGCCACC ATGAAAACGATTTCCGTTGTTA-3' } \\
5^{\prime} \text {-CCCGTCGACACGGATTTATATTCACAGCAACA- } 3^{\prime}\end{array}$ & 992 & $\begin{array}{l}\text { pBKRSV-B7R } \\
\text { pcDNA-B7R }\end{array}$ \\
\hline F8L & $\begin{array}{c}5^{\prime} \text {-CCCGGCTAGCCGCCACC ATGTCACAACAACTATCTCCTA-3' } \\
5^{\prime} \text {-CCCGTCGACAATCTAGTTTTGTTTTTCTCG-3' }\end{array}$ & 944 & $\begin{array}{l}\text { pBKRSV-F8L } \\
\text { pcDNA-F8L }\end{array}$ \\
\hline M1R & $\begin{array}{l}5^{\prime} \text {-CCCCGCTAGCCGCCACC ATGGGTGCCGCGGCAAG-3' } \\
5^{\prime} \text {-CCCCGTCGACTTCAGTTTTGTATATCCGTGGTAGCAAT-3' }\end{array}$ & 781 & $\begin{array}{l}\text { pBKRSV-M1R } \\
\text { pcDNA-M1R }\end{array}$ \\
\hline
\end{tabular}

AsuNHI (GCTAGC) and SalI (GTCGAC) sites for cloning sequence are boldfaced; initiation and stop codons are underlined; a Kozak sequence added between the initiation codon and protein sequence is italicized.

TABLE 2: Comparison of VARV, VACV, CPXV, MPXV, and ECTV proteins.

\begin{tabular}{|c|c|c|c|c|c|c|}
\hline \multirow{2}{*}{ VARV protein } & \multirow{2}{*}{ Length (aa)/number of epitopes } & \multicolumn{5}{|c|}{ Homology, \%/number of coincident B-cell epitopes ${ }^{*}$} \\
\hline & & VARV $^{* *}$ & $\mathrm{VACV}^{* *}$ & $\mathrm{CPXV}^{* *}$ & $\mathrm{MPXV}^{* *}$ & $\mathrm{ECTV}^{* *}$ \\
\hline A30L & $110 / 5$ & $99.1-100.0 / 5$ & $96.4-97.3 / 4$ & $97.3-98.2 / 4$ & $92.7-93.6 / 3-4$ & $95.6 / 3$ \\
\hline A36R & $184 / 6$ & $100.0 / 6$ & $93.6-94.1 / 3$ & $92.0-95.2 / 3-4$ & $89.8 / 3$ & $85.3 / 1$ \\
\hline B7R & $317 / 7$ & $99.7-100.0 / 6-7$ & $92.7-93.1 / 3-4$ & $92.4-94.0 / 4-5$ & $92.7 / 4$ & $91.1 / 3$ \\
\hline $\mathrm{F} 8 \mathrm{~L}$ & $304 / 8$ & $100.0 / 8$ & $95.7-96.1 / 6-7$ & $93.4-95.1 / 6-7$ & $92.8 / 6$ & $93.8 / 6$ \\
\hline M1R & $250 / 8$ & $99.6-100.0 / 7-8$ & $99.2-99.6 / 7$ & $99.2 / 7-8$ & $99.2 / 8$ & $98.4 / 7$ \\
\hline
\end{tabular}

* $\%$ of homology and number of coincident epitopes were computed with respect to VARV strain India-1967 proteins.

${ }^{* *}$ Data on several strains.

computed using the ADEPT version 2.1 program [34]. Subsequent analysis has demonstrated that some VARV proteins insignificantly differ in their potential antigenic determinants from their VACV orthologs, namely, A30L and M1R, in one potential antigenic determinant and F8L, in two determinants, whereas the products of VARV genes A36R and $\mathrm{B} 7 \mathrm{R}$ display considerable distinctions in the number of their potential antigenic determinants from their orthologs of other orthopoxvirus species (Table 2). Distribution of such discrepancies in amino acid sequence of A36R protein is given as an example in Figure 1.

\subsection{The Optimal Method for DNA Vaccine Administration} Depends on the Used Promoter for Eukaryotic Expression. The intraperitoneal immunization of BALB/c mice with the DNA vaccines based on individual VARV genes $\mathrm{A} 4 \mathrm{~L}$, A30L, A36R, B7R, F8L, I5R, M1R, and M4R demonstrated that the plasmid pBKRSV-F8L had the most pronounced immunogenic effect [35]. It is known that the administration route and used eukaryotic promoter can influence the DNA vaccine efficacy [36]. Therefore, we used the plasmids pBKRSV-F8L and pcDNA-F8L for immunizing mice at a dose of $100 \mu \mathrm{g} /$ mouse using different administration routes, namely, intraperitoneal, intramuscular, intracutaneous, or subcutaneous. In two groups for negative and positive control, the mice were intraperitoneally immunized with physiological saline solution and VACV at a dose of $10^{6} \mathrm{PFU}$ per mouse, respectively. All preparations were applied three times with 3-week intervals to intraperitoneally challenge of 10-11 mice in each group with ECTV-K1 at a dose of $10 \mathrm{LD}_{50}$ 3 weeks after the last immunization. The best results were obtained for intracutaneous immunization with the DNA vaccines pcDNA-F8L (40\% of survivals; Figure 2(a)) and intraperitoneal administration of the DNA vaccine pBKRSVF8L (45\% of survivals; Figure 2(b)). The combinations pcDNA-F8L/intraperitoneally, pcDNA-F8L/subcutaneously, pBKRSV-F8L/intracutaneously, or pBKRSV-F8L/subcutaneously gave considerably lower protection levels, namely, $9,18,18$, and $9 \%$, respectively. No survived animals were recorded in the groups intramuscularly immunized with the DNA vaccines $\mathrm{pBKRSV}-\mathrm{F} 8 \mathrm{~L}$ or $\mathrm{pcDNA}-\mathrm{F} 8 \mathrm{~L}$.

3.3. Immunization with Individual IMV Genes Provides a Partial Protection. Several groups were intracutaneously immunized at a dose of $100 \mu \mathrm{g} /$ mouse with the single-gene DNA vaccines pcDNA-A30L, pcDNA-F8L, or pcDNA-M1R as well as the control plasmid pcDNA and intraperitoneally with VACV at a dose of $10^{6} \mathrm{PFU} /$ mouse as negative and positive controls, respectively. The vaccines were administered three times with 3-week intervals to intraperitoneally challenge of 30-32 mice in each group with ECTV-K1 at a dose of $10 \mathrm{LD}_{50}$. This experiment has demonstrated that the VARV (IMV) genes F8L, A30L, and M1R within the vector pcDNA administered intracutaneously provide 40,33 , and 
VARV_IND
VARV_GAR
VARV_BSH
VACV_COP
VACV_WR
VACV_ANK
CPXV_BRT
CPXV_GRI
MPXV_ZAI
MPXV_USA
ECTV_MOS
ECTV_NAV

VARV_IND

VARV_GAR

VARV BSH

VACV_COP

VACV_WR

VACV_ANK

CPXV_BRT

CPXV_GRI

MPXV_ZAI

MPXV_USA

ECTV_MOS

ECTV_NAV

VARV_IND

VARV_GAR

VARV_BSH

VACV_COP

VACV_WR

VACV_ANK

CPXV_BRT

CPXV_GRI

MPXV_ZAI

MPXV_USA

ECTV_MOS

ECTV_NAV

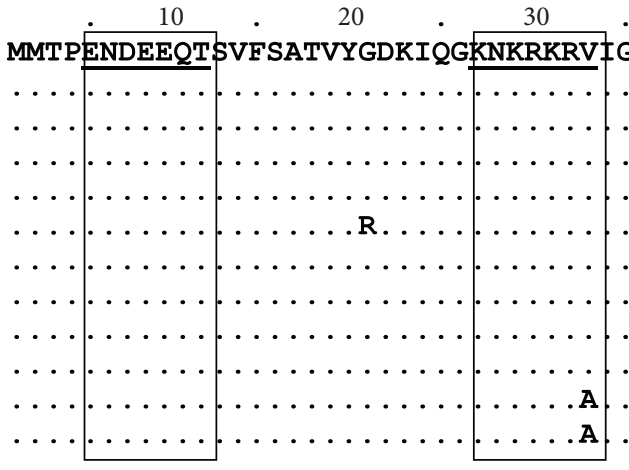

40

50

60

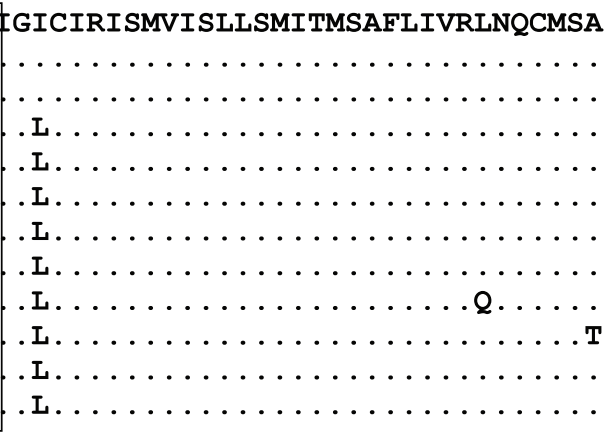

70

80

90

100

110

120

130

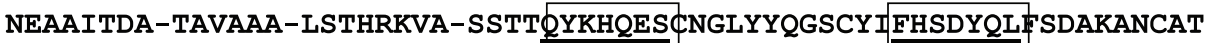

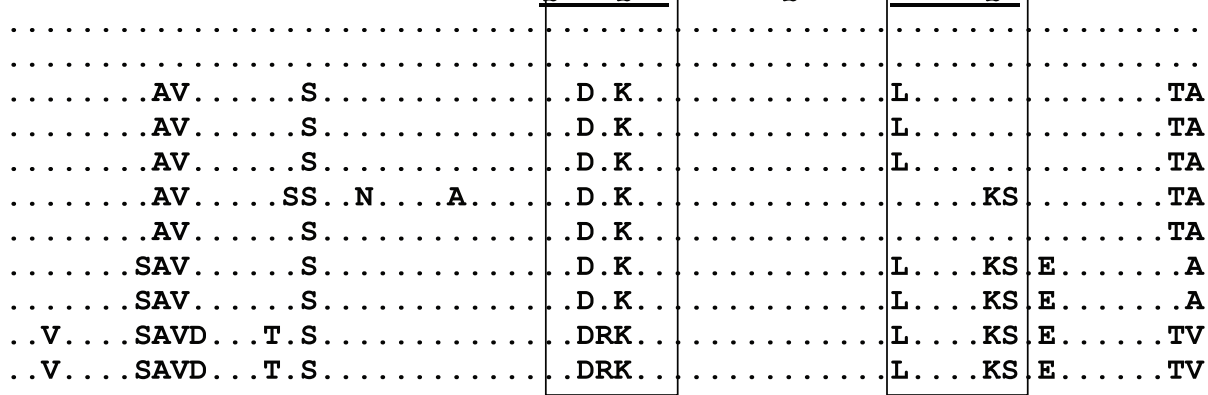

$140 \quad 150 \quad$. $\quad 160 \quad$. $170 \quad . \quad 180$

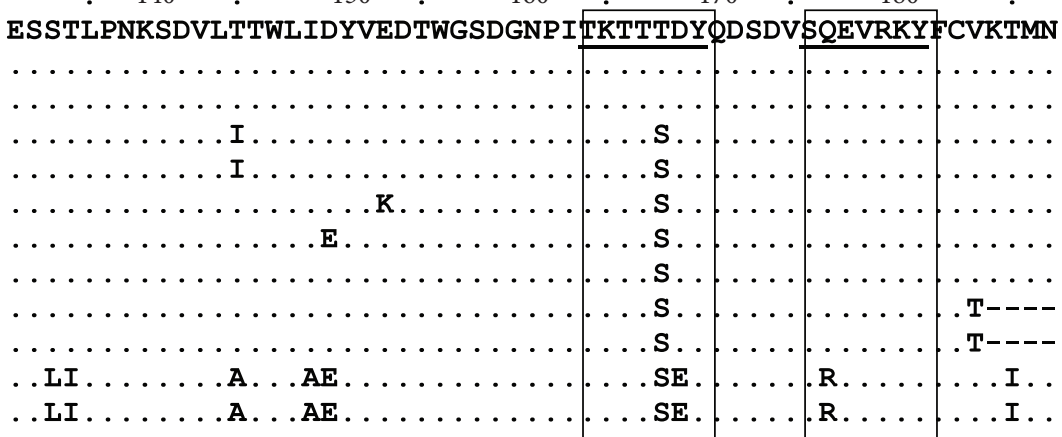

FIGURE 1: Comparison of the amino acid sequence of the VARV A36R protein with the corresponding VACV, CPXV, MPXV, and ECTV orthologs. Identical amino acids in the compared sequence of virus genomes relative to the VARV strain India-1967 are denoted with dots and deletions, with dashes. The amino acid positions in analyzed segment are shown above the amino acid sequence. Potential VARV antigenic determinants are underlined and framed. The strains VARV_IND, VARV_GAR, VARV_BSH, VACV_COP, VACV_WR, VACV_ANK, CPXV_BRT, CPXV_GRI, MPXV_ZAI, MPXV_USA, and ECTV_MOS have the following PubMed accession numbers: X69198, Y16780, L22579, M35027, AY243312, U94848, AF482758, X94355, AF380138, DQ011157, and AF012825. The ECTV_NAV amino acid sequence was taken from http://www.sanger.ac.uk/.

$25 \%$ protection, respectively, from the challenge with $10 \mathrm{LD}_{50}$ of ECTV (Figure 3). The observed protective efficacies of the DNA vaccines involving individual VARV (IMV) genes correlated with the virus-neutralizing activities of the sera: VARV genes F8L, A30L, and M1R within the vector pcDNA administered intracutaneously induced 57.0 \pm 3.6, $52.0 \pm$ 4.0 and $40.0 \pm 2.5 \%$ level of neutralizing antibodies against VACV, respectively.

3.4. The Polyvalent DNA Vaccine Involving the VARV Genes A30L, M1R, F8L, A36R, and B7R Induces Production of High Titers of VARV-Neutralizing Antibodies and Provides a Complete Protection against the Challenge with a Lethal ECTV Dose. Based on the results of previous studies $[15,17$, 18 , 37], the following immunization schemes for the animal groups were used to assess the efficacy of polyvalent DNA vaccine: (1) intracutaneous immunization with a mixture of the five plasmids pcDNA-A30L, pcDNA-F8L, pcDNA-M1R, pcDNA-A36R, and pcDNA-B7R, $50 \mu \mathrm{g}$ each (a total dose of $250 \mu \mathrm{g} /$ mouse); (2) intraperitoneal immunization with a mixture of the plasmids pBKRSV-A30L, pBKRSV-F8L, pBKRSVM1R, pBKRSV-A36R, and pBKRSV-B7R, $50 \mu \mathrm{g}$ each (a total dose of $250 \mu \mathrm{g} /$ mouse); (3) intracutaneous immunization with the plasmid pcDNA at a dose of $250 \mu \mathrm{g} / \mathrm{mouse}$ (negative 


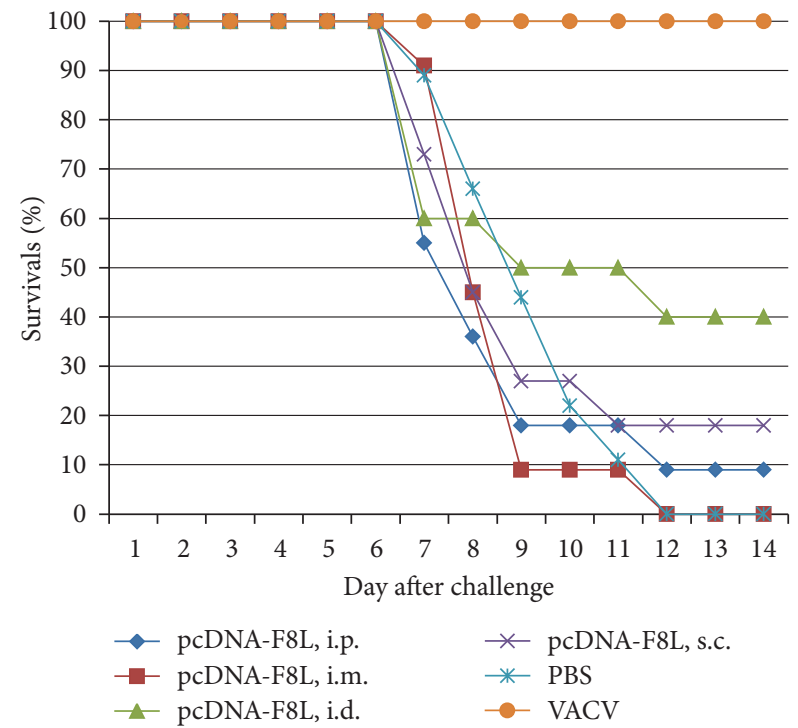

(a)

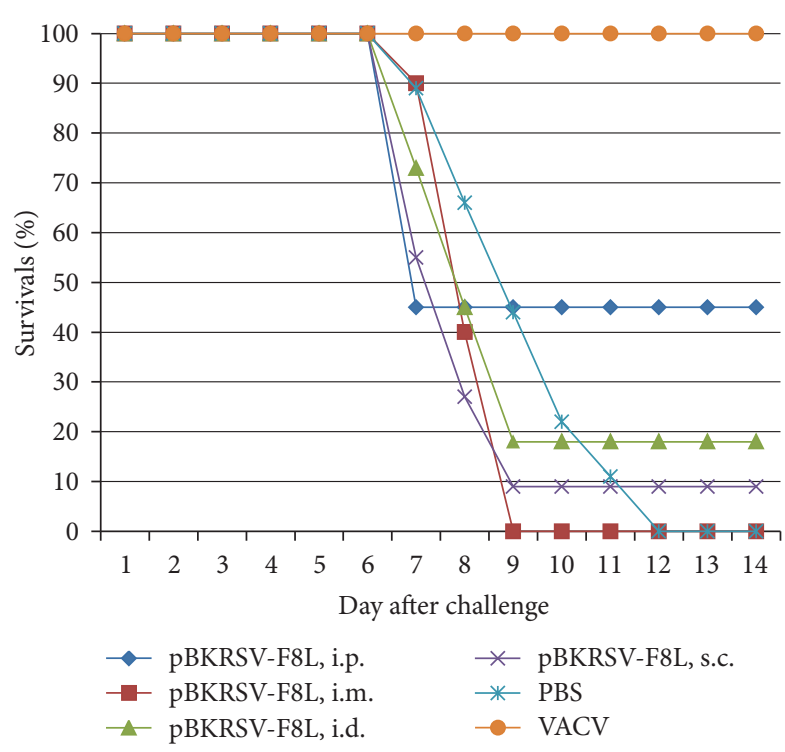

(b)

FIGURE 2: The survival rates of BALB/c mice after triple intraperitoneal (i/p), intramuscular (i/m), intracutaneous (i/c), or subcutaneous (s/c) immunization with (a) pcDNA-F8L or (b) pBKRSV-F8L and intraperitoneal challenge with ectromelia virus strain $\mathrm{K}_{1}$ at a dose of $10 \mathrm{LD}_{50}$. 10-11 mice in each group were challenged 3 weeks after the last immunization.

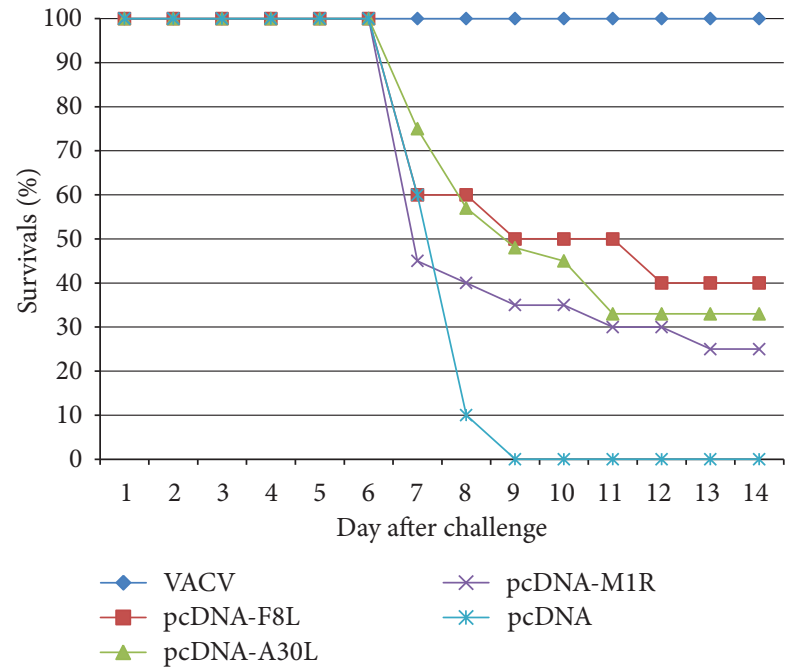

FIgURE 3: The survival rates of BALB/c mice after triple intracutaneous immunization with the DNA vaccines pcDNA-A30L, pcDNA-F8L, or pcDNA-M1R and intraperitoneal challenge with ectromelia virus strain $\mathrm{K} 1$ at a dose of $10 \mathrm{LD}_{50} .30-32$ mice in each group were challenged 3 weeks after the last immunization.

control no. 1); (4) intraperitoneal immunization with the plasmid pBKRSV at a dose of $250 \mu \mathrm{g} /$ mouse (negative control no. 2) and (5) intraperitoneal immunization with VACV at a dose of $10^{6} \mathrm{PFU} /$ mouse (positive control). The vaccines were administered with 3 -week intervals; 3 weeks after the last immunization, 30 mice in each group were intraperitoneally challenged with ECTV-K1 at a dose of $10 \mathrm{LD}_{50}$.

The immunizations with both variants of the polyvalent DNA vaccine induced production of the VACV-neutralizing

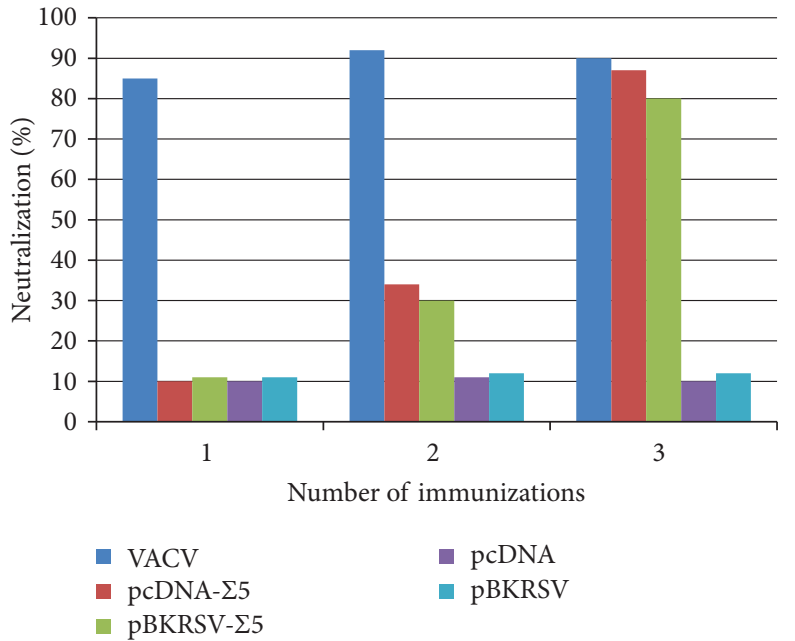

FIgURE 4: Dynamics of the level of virus-neutralizing antibodies during intracutaneous immunization of $\mathrm{BALB} / \mathrm{c}$ mice with the polyvalent DNA vaccine pcDNA-A30L, pcDNA-F8L, pcDNA-M1R, pcDNA-A36R, and pcDNA-B7R (pcDNA- $\Sigma 5$ ); intraperitoneally with the polyvalent DNA vaccine pBKRSV-A30L, pBKRSV-F8L, pBKRSV-M1R, pBKRSV-A36R, and pBKRSV-B7R (pBKRSV- 5 5); or intraperitoneally with VACV detected by VACV neutralization in Vero cell culture. Mean values of the neutralization (\%) for serum dilution of $1: 100 \pm$ standard deviations are shown. Numbers 1, 2, and 3 indicates the number of immunizations.

antibodies (Figure 4). Note that the level of neutralizing antibodies after the third immunization with DNA vaccines was comparable with the level of neutralizing antibodies after immunization with the live VACV, $90.0 \pm 5.0 \%$ (the data are shown for the mouse serum titer of 1/100). The polyvalent 


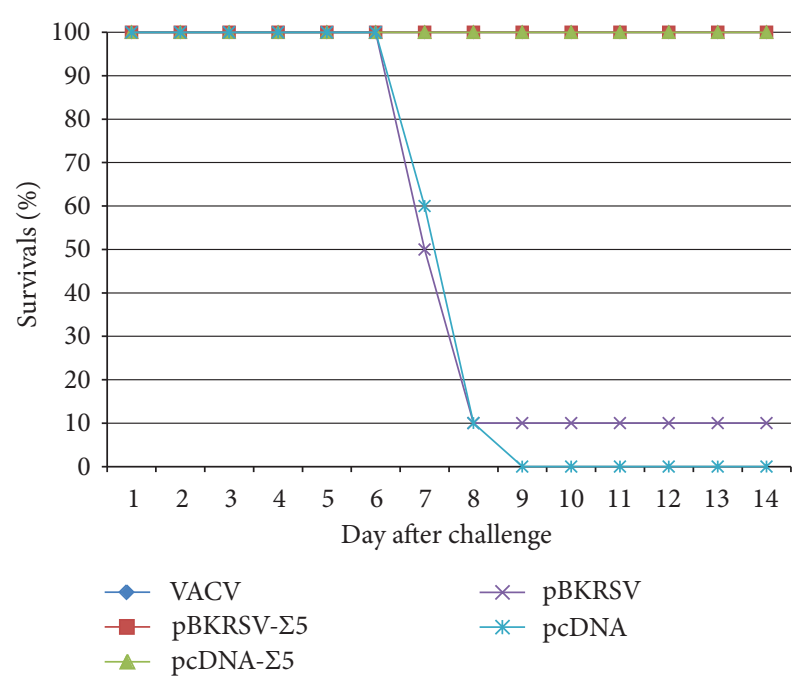

FIgURE 5: The survival rates of $\mathrm{BALB} / \mathrm{c}$ mice after triple intracutaneous immunization with the polyvalent DNA vaccine pcDNAA30L, pcDNA-F8L, pcDNA-M1R, pcDNA-A36R, and pcDNA-B7R (pcDNA- 55 ) or triple intraperitoneal immunization with the polyvalent DNA vaccine pBKRSV-A30L, pBKRSV-F8L, pBKRSV-M1R, pBKRSV-A36R, and pBKRSV-B7R (pBKRSV- 55 ) and intraperitoneal challenge with ectromelia virus strain $\mathrm{K} 1$ at a dose of 10 $\mathrm{LD}_{50} .30$ mice in each group were challenged 3 weeks after the last immunization.

DNA vaccine based on the vector pcDNA induced a similar level of neutralizing antibodies $(87.0 \pm 4.0 \%)$ as compared with the vaccine based on the vector pBKRSV $(80.0 \pm 2.5 \%)$.

When assessing the protective efficacy, the immunized mice were challenged with ECTV-K1 at a dose of $10 \mathrm{LD}_{50}$ with subsequent observation for 14 days (Figure 5). In negative control groups, $90 \%$ of the mice immunized with pBKRSV and $100 \%$ of the animals immunized with pcDNA died by the end of the observation period. No mice died by the end of the observation period in the group immunized with live VACV. Both variants of the polyvalent DNA vaccine involving the VARV genes A30L, F8L, M1R, A36R, and B7R also provided a complete protection of the mice from the challenge with a lethal ECTV dose.

\section{Discussion}

It is known that only those persons who had smallpox acquire almost a lifelong immunity to this disease, whereas those who were vaccinated with VACV require repeated immunizations with a certain periodicity to maintain their antismallpox immune status $[1,3]$. A reliable protection against orthopoxvirus infection implies a concurrent induction of the immune responses to two infective forms of the virus, intracellular and extracellular virions, which is achievable only when using the immunogens of intracellular virion surface membrane and extracellular virus envelope within one vaccine $[15,17,18,37]$.

A high potential of the VARV genes A30L, M1R, and F8L, encoding intracellular virion surface membrane proteins, and the genes A36R and B7R, encoding the extracellular virus envelope proteins, in development of the anti-smallpox DNA vaccines has been recently shown for their VACV orthologs in protection of mice against VACV infection [16, 37]. At the first stage of the work, we compared the structures of these immunodominant proteins of the orthopoxviruses pathogenic for humans (VARV, MPXV, CPXV, VACV) with the same proteins of ECTV, nonpathogenic for humans but highly pathogenic for mice. We have used a previously designed ADEPT software program which localizes potential B cell epitopes in analyzed proteins [34]. It uses a matrix of amino acid values to calculate moving average along the sequence. The matrix contains a value for each amino acid which shows how much an amino acid contributes to the region being potential $\mathrm{B}$ cell epitope. The matrix was created by running the program in learning mode on the database of well-known proteins. In the case of orthopoxviral proteins, these potential epitopes have not been confirmed experimentally but were analyzed using ADEPT program for understanding that the DNA vaccine involving the VARV genes should be more efficacious as compared with the vaccine based on VACV genes in inducing the protective immunity of humans to smallpox. Despite that orthopoxviruses differ in the number (Table 2) and location (Figure 1) of their potential antigenic determinants, immunization of animals with a representative of one of the species induces a cross-immune response provided for protection against other orthopoxvirus species [1]. Although this protection is suboptimal, it is still possible to conduct preclinical trials of the developed vaccines against smallpox using the models of small laboratory animals and the orthopoxviruses pathogenic for them. The most highly pathogenic virus for mice is ECTV, which we correspondingly selected for assessing a protective efficacy of the developed DNA vaccine.

It is known that the efficacy of a DNA vaccine depends to a considerable degree on various parameters, such as the used antigen, structure of vector plasmid, promoter for eukaryotic expression of the target antigen, administration route, amount of plasmid, and number of immunizations, since all these factors influence the strength and quality of the induced immune response [36]. Correspondingly, aiming to increase the efficacy of the elaborated DNA vaccine, we have analyzed the combination of administration route and used eukaryotic promoter by the example of VARV F8L gene to select the optimal variant providing for induction of the most pronounced protective immune response to orthopoxvirus infection. This has allowed us to discover that the DNA vaccine carrying the promoter from RSV (pBKRSV) is the most efficient in the case of intraperitoneal administration ( $45 \%$ of survivals), while the DNA vaccine with the CMV promoter (pcDNA) and the same gene of VARV immunodominant protein is the most efficient in the case of intracutaneous administration (40\% of survivals). Other combinations of rate immunization and type of DNA vaccine gave no more than $18 \%$ of survivals. This allows us to give a conclusion about the reliability of obtained results. It has been earlier repeatedly demonstrated that the efficiencies of these two promoters in inducing the immune response to the protein product of a target gene depends on particular experimental conditions. For example, it has been shown in 
the work on designing the DNA vaccine against influenza virus based on the virus nucleoprotein gene that the CMV promoter provides a stronger humoral response in the case of an intracutaneous administration as compared with the RSV promoter, although both promoters induce equal immune responses when the DNA vaccine is administered intramuscularly [38].

At the next stage of our work, we have demonstrated that the DNA vaccines based on the individual genes M1R, A30L, or F8L, encoding VARV intracellular virion surface membrane proteins, under the control of CMV promoter being intracutaneously administered induce production of VACV-neutralizing antibodies and provide for a partial mouse protection against ECTV challenge at a dose of 10 $\mathrm{LD}_{50}$ (Figure 3). Earlier Hooper et al. [16] have demonstrated that the DNA vaccines based on two EEV proteins, encoded by A33R and B5R VACV (orthologs to A36R and B7R VARV), provide for $80 \%$ mouse protection against VACV WR challenge at a dose of $12.5 \mathrm{LD}_{50}$. At the same time combination of two IMV proteins, encoded by A27L and L1R, and two EEV proteins, encoded by A33R and B5R VACV (orthologs to A30L, M1R, A36R, and B7R VARV), provide for $100 \%$ mouse protection against VACV WR challenge at a dose of $12.5 \mathrm{LD}_{50}$ [16].

Using a combined variant of the DNA vaccine involving five plasmids carrying the genes M1R, A30L, and F8L of intracellular virion surface membrane proteins as well as A36R and B7R of the extracellular virus envelope proteins, it has been shown that both variants of the DNA vaccine-the variant with the vector pBKRSV administered intraperitoneally and the variant with the vector pcDNA administered intracutaneously-induce production of a high titers of VACV-neutralizing antibodies similar to the live VACV vaccine (Figure 4) and provide a complete protection of the animals against a lethal dose of highly pathogenic ECTV (Figure 5). The considerable differences of the used VARV antigens from their ECTV orthologs in both the number and localization of their potential antigenic determinants (Table 2) as well as the observed protective effect to lethal ECTV challenge after immunization with the elaborated combined DNA vaccines suggest that such vaccine should be efficient in immunization of humans against smallpox. Using of the developed DNA vaccine for prime-immunization of humans against smallpox with following boost-immunization with VACV will allow avoid a side effects characteristic to classic alive smallpox vaccine used alone [2].

\section{References}

[1] F. Fenner, D. A. Henderson, I. Arita, Z. Jezek, and I. D. Ladnyi, Smallpox and Its Eradication, World Health Organization, Geneva, Switzerland, 1988.

[2] F. Fenner, "Risks and benefits of vaccinia vaccine use in the worldwide smallpox eradication campaign," Research in Virology, vol. 140, pp. 465-466, 1989.

[3] S. N. Shchelkunov, S. S. Marennikova, and R. W. Moyer, Orthopoxviruses Pathogenic for Humans, Springer, New York, NY, USA, 2005.

[4] S. N. Shchelkunov, "How long ago did smallpox virus emerge?" Archives of Virology, vol. 154, no. 12, pp. 1865-1871, 2009.
[5] S. N. Shchelkunov, "Emergence and reemergence of smallpox: the need in development of a new generation smallpox vaccine," Vaccine, vol. 29, supplement 4, pp. D49-D53, 2011.

[6] J. Stephenson, "Monkeypox outbreak a reminder of emerging infections vulnerabilities," Journal of the American Medical Association, vol. 290, no. 1, pp. 23-24, 2003.

[7] S. Lewis-Jones, "Zoonotic poxvirus infections in humans," Current Opinion in Infectious Diseases, vol. 17, no. 2, pp. 81-89, 2004.

[8] H. Campe, P. Zimmermann, K. Glos et al., "Cowpox virus transmission from pet rats to humans, Germany," Emerging Infectious Diseases, vol. 15, no. 5, pp. 777-780, 2009.

[9] L. Ninove, Y. Domart, C. Vervel et al., "Cowpox virus transmission from pet rats to humans, France," Emerging Infectious Diseases, vol. 15, no. 5, pp. 781-784, 2009.

[10] A. W. Rimoin, P. M. Mulembakani, S. C. Johnston et al., "Major increase in human monkeypox incidence 30 years after smallpox vaccination campaigns cease in the Democratic Republic of Congo," Proceedings of the National Academy of Sciences of the United States of America, vol. 107, no. 37, pp. 16262-16267, 2010.

[11] R. G. Webster, E. F. Fynan, J. C. Santoro, and H. Robinson, "Protection of ferrets against influenza challenge with a DNA vaccine to the haemagglutinin," Vaccine, vol. 12, no. 16, pp. 1495-1498, 1994.

[12] J. W. Hooper, K. I. Kamrud, F. Elgh, D. Custer, and C. S. Schmaljohn, "DNA vaccination with hantavirus $M$ segment elicits neutralizing antibodies and protects against seoul virus infection," Virology, vol. 255, no. 2, pp. 269-278, 1999.

[13] J. Riemenschneider, A. Garrison, J. Geisbert et al., "Comparison of individual and combination DNA vaccines for B. anthracis, Ebola virus, Marburg virus and Venezuelan equine encephalitis virus," Vaccine, vol. 21, no. 25-26, pp. 4071-4080, 2003.

[14] S. N. Shchelkunov, "Functional organization of variola major and vaccinia virus genomes," Virus Genes, vol. 10, no. 1, pp. 53-71, 1995.

[15] J. W. Hooper, D. M. Custer, C. S. Schmaljohn, and A. L. Schmaljohn, "DNA vaccination with vaccinia virus L1R and A33R genes protects mice against a lethal poxvirus challenge," Virology, vol. 266, no. 2, pp. 329-339, 2000.

[16] J. W. Hooper, D. M. Custer, and E. Thompson, "Four-genecombination DNA vaccine protects mice against a lethal vaccinia virus challenge and elicits appropriate antibody responses in nonhuman primates," Virology, vol. 306, no. 1, pp. 181-195, 2003.

[17] P. Sakhatskyy, S. Wang, T. H. W. Chou, and S. Lu, "Immunogenicity and protection efficacy of monovalent and polyvalent poxvirus vaccines that include the D8 antigen," Virology, vol. 355, no. 2, pp. 164-174, 2006.

[18] J. W. Hooper, E. Thompson, C. Wilhelmsen et al., "Smallpox DNA vaccine protects nonhuman primates against lethal monkeypox," Journal of Virology, vol. 78, no. 9, pp. 4433-4443, 2004.

[19] J. M. Heraud, Y. Edghill-Smith, V. Ayala et al., "Subunit recombinant vaccine protects against monkeypox," Journal of Immunology, vol. 177, no. 4, pp. 2552-2564, 2006.

[20] P. Sakhatskyy, S. Wang, C. Zhang, T. H. Chou, M. Kishko, and S. $\mathrm{Lu}$, "Immunogenicity and protection efficacy of subunit-based smallpox vaccines using variola major antigens," Virology, vol. 371, no. 1, pp. 98-107, 2008.

[21] S. N. Shchelkunov, S. S. Marennikova, A. V. Totmenin et al., "Creation of a clone library of fragments from the natural variola virus and study of the structural and functional organization 
of viral genes from a circle of hosts," Doklady Akademii Nauk SSSR, vol. 321, no. 2, pp. 402-406, 1991.

[22] S. N. Shchelkunov, S. M. Resenchuk, A. V. Totmenin, V. M. Blinov, S. S. Marennikova, and L. S. Sandakhchiev, "Comparison of the genetic maps of variola and vaccinia viruses," The FEBS Letters, vol. 327, no. 3, pp. 321-324, 1993.

[23] R. F. Massung, L. I. Liu, J. Qi et al., "Analysis of the complete genome of smallpox variola major virus strain Bangladesh1975," Virology, vol. 201, no. 2, pp. 215-240, 1994.

[24] S. N. Shchelkunov, A. V. Totmenin, V. N. Loparev et al., "Alastrim smallpox variola minor virus genome DNA sequences," Virology, vol. 266, no. 2, pp. 361-386, 2000.

[25] S. N. Shchelkunov, A. V. Totmenin, I. V. Babkin et al., "Human monkeypox and smallpox viruses: genomic comparison," The FEBS Letters, vol. 509, no. 1, pp. 66-70, 2001.

[26] A. M. Likos, S. A. Sammons, V. A. Olson et al., "A tale of two clades: monkeypox viruses," Journal of General Virology, vol. 86, no. 10, pp. 2661-2672, 2005.

[27] S. N. Shchelkunov, P. F. Safronov, A. V. Totmenin et al., "The genomic sequence analysis of the left and right speciesspecific terminal region of a cowpox virus strain reveals unique sequences and a cluster of intact ORFs for immunomodulatory and host range proteins," Virology, vol. 243, no. 2, pp. 432-460, 1998.

[28] F. Q. Hu, C. A. Smith, and D. J. Pickup, "Cowpox virus contains two copies of an early gene encoding a soluble secreted form of the type II TNF receptor," Virology, vol. 204, no. 1, pp. 343-356, 1994.

[29] S. J. Goebel, G. P. Johnson, M. E. Perkus, S. W. Davis, J. P. Winslow, and E. Paoletti, "The complete DNA sequence of vaccinia virus," Virology, vol. 179, no. 1, pp. 247-566, 1990.

[30] G. Antoine, F. Scheiflinger, F. Dorner, and F. G. Falkner, "The complete genomic sequence of the modified vaccinia Ankara strain: comparison with other orthopoxviruses," Virology, vol. 244, no. 2, pp. 365-396, 1998.

[31] B. M. Baroudy and B. Moss, "Sequence homologies of diverse length tandem repetitions near ends of vaccinia virus genome suggest unequal crossing over," Nucleic Acids Research, vol. 10, no. 18 , pp. 5673-5679, 1982.

[32] K. Mossman, C. Upton, R. M. L. Buller, and G. McFadden, "Species specificity of ectromelia virus and vaccinia virus interferon- $\gamma$ binding proteins," Virology, vol. 208, no. 2, pp. 762-769, 1995.

[33] E. J. Dick Jr., C. L. Kittell, H. Meyer et al., "Mousepox outbreak in a laboratory mouse colony," Laboratory Animal Science, vol. 46, no. 6, pp. 602-611, 1996.

[34] A. Z. Maksyutov and E. S. Zagrebelnaya, "ADEPT: a computer program for prediction of protein antigenic determinants," Computer Applications in the Biosciences, vol. 9, no. 3, pp. 291-297, 1993.

[35] R. A. Maksyutov, I. N. Babkina, A. E. Nesterov, and S. N. Shchelkunov, "Creation of a candidate DNA vaccine against human orthopoxvirus infections," Biotekhnologiya, vol. 4, pp. 23-30, 2006.

[36] N. A. Doria-Rose and N. L. Haigwood, "DNA vaccine strategies: candidates for immune modulation and immunization regimens," Methods, vol. 31, no. 3, pp. 207-216, 2003.

[37] D. J. Pulford, A. Gates, S. H. Bridge, J. H. Robinson, and D. Ulaeto, "Differential efficacy of vaccinia virus envelope proteins administered by DNA immunisation in protection of BALB/c mice from a lethal intranasal poxvirus challenge," Vaccine, vol. 22, no. 25-26, pp. 3358-3366, 2004.
[38] E. Raz, D. A. Carson, S. E. Parker et al., "Intradermal gene immunization: the possible role of DNA uptake in the induction of cellular immunity to viruses," Proceedings of the National Academy of Sciences of the United States of America, vol. 91, no. 20, pp. 9519-9523, 1994. 

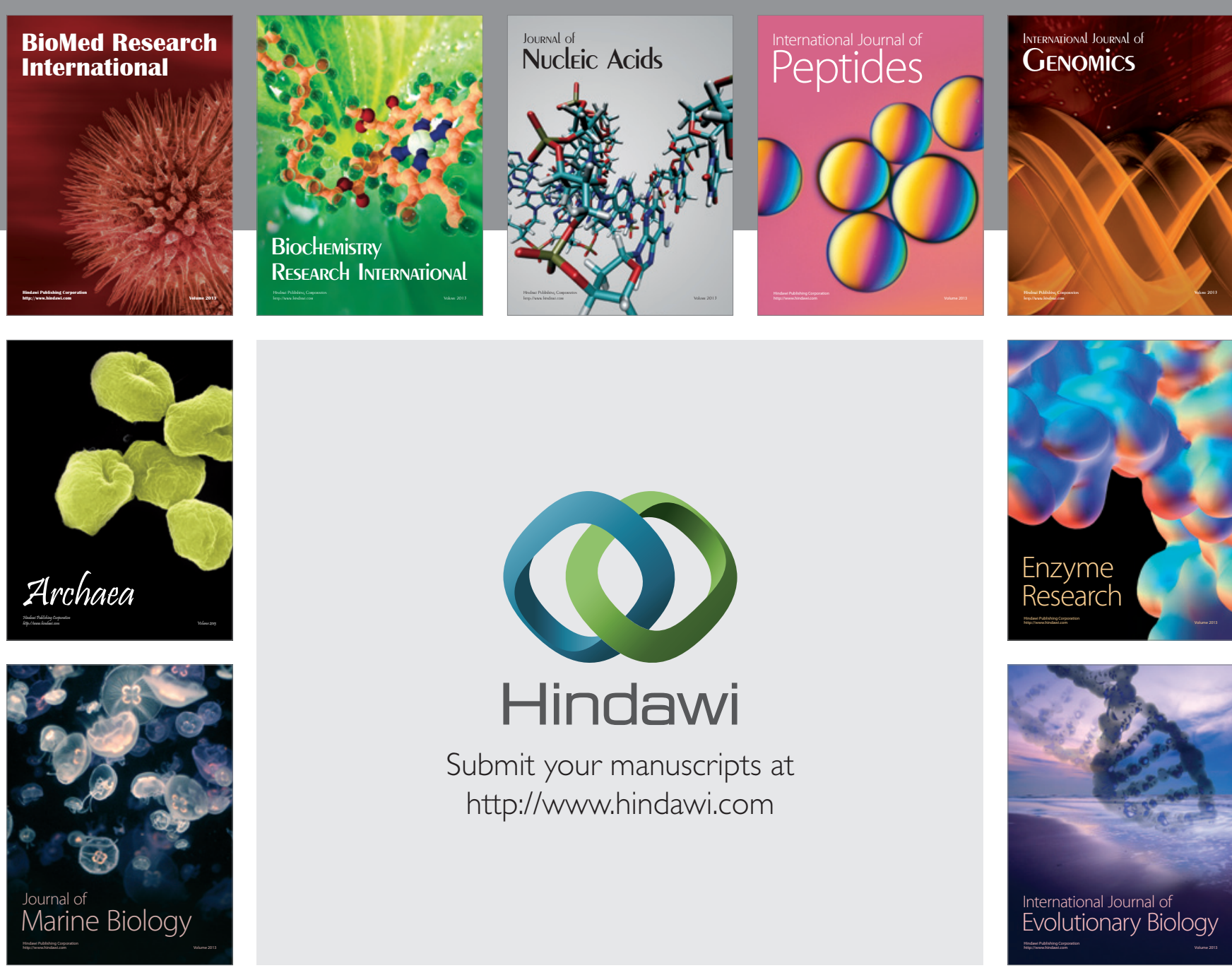

Submit your manuscripts at

http://www.hindawi.com
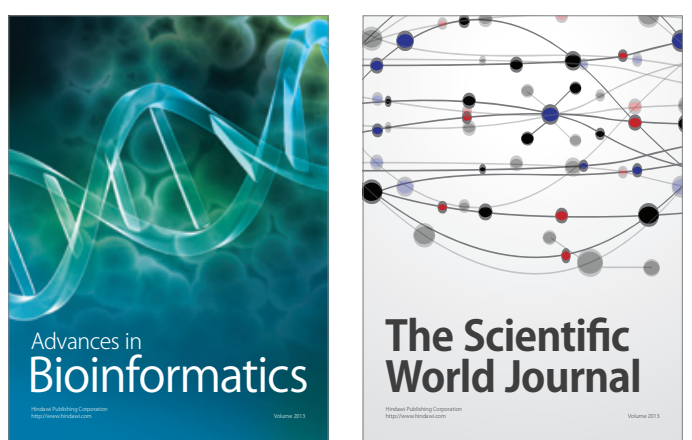

The Scientific World Journal

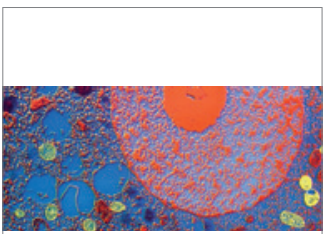

ISRN

Cell Biology

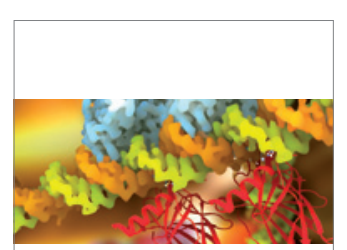

ISRN

Molecular Biology
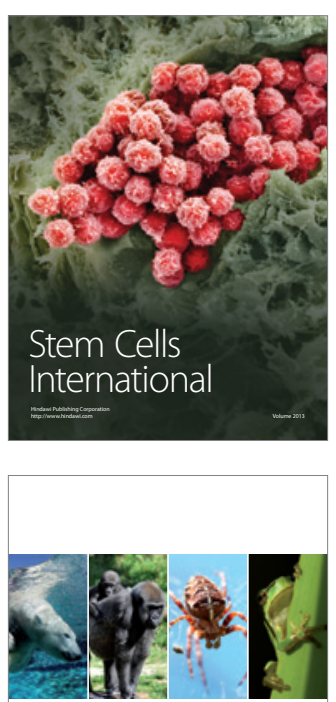

ISRN

Zoology

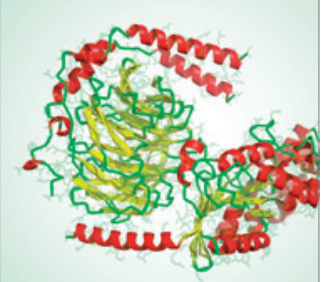

Signal Transduction

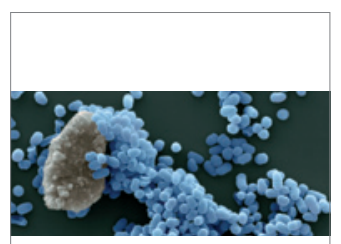

ISRN

Biotechnology
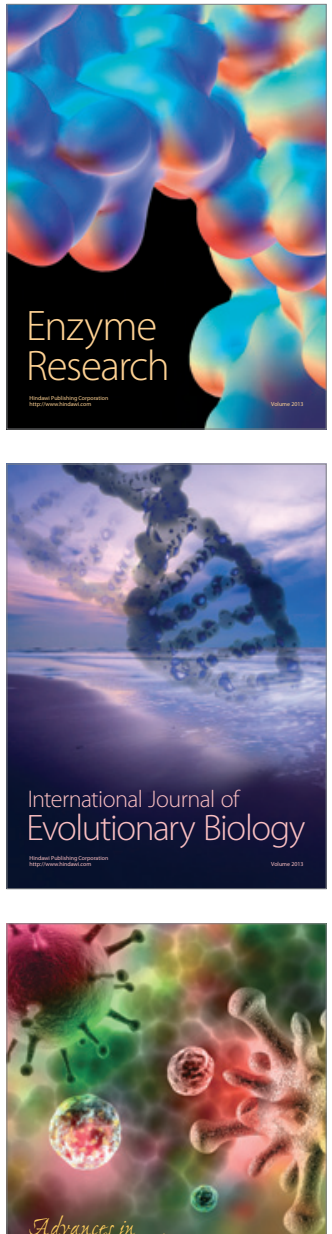

\section{Virology}

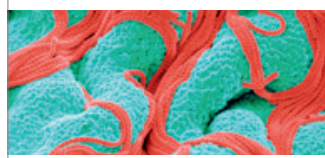

ISRN

Microbiology 